\title{
Microstructure Investigation of Hydrous Ruthenium Oxides Coatings with Carbon-Nanotube Additive by Cathodic Deposition Method
}

\author{
Yuli Lin and H.-S Hwang
}

The Institute of Engineering Science

Chung Hua University, Hsinchu, 300 Taiwan ROC

Supercapacitors have many advantages using in electrical devices for their larger capacitance high power density and long cycle life. In general, supercapacitors can be classified into two categories, namely, pseudo-capacitors and double-layer capacitors. The fomer stores electrical charges in electrode surface region by faradaic reaction. While, in the latter, electrical charges are stored ate the double-layer formed at electrode/electrolyte interface. Various methods have been utilized to manufacture electrode including cyclic voltammetric method 、 sol-gel method 、 anodizing 、 cathodic deposition method and etc.[1].

In this study, hydrous ruthenium oxide with adding carbon nanotube was codeposited on $\mathrm{Ti}$ substrate by cathodic deposition method. Ti substrate was first cleaned thoroughly by acetone and followed by chemical etching of $5 \% \mathrm{HF}$ for 5 minutes and $50 \% \mathrm{HCl}$ for 15 minutes. These processes not only will produce many intensive microvoids which increase the mechanical locking of the coating layer [2]. The purpose of adding carbon nanotube is to increase the deposition efficiency of hydrous ruthenium oxide coating during deposition. The concentration of carbon nanotube added in the deposition process is $0.25 \mathrm{wt} \%$. The specimen was then immersed into the deposition bath for 5 minutes. The electrical capacity characteristics of coating were then examined by cyclic voltammetry. The microstructure of hydrous ruthenium oxide coating was also observed by scanning electron microscopy.

Figure 1(a) and (b) show the microstructure of hydrous ruthenium oxide coating with adding carbon nanotube. It clearly demonstrates that microvoid type of pattern remains after hydrous ruthenium oxide coating was deposited on Ti substrate. The capacitance of this specimen was measured about $258.9 \mathrm{~F} / \mathrm{g}$. Figure 2 reveals the microstructure at even higher magnification. It was found that very tiny hydrous ruthenium oxide particles were deposited on Ti substrate. Due to short deposition time, small amount of carbon nanotube was co-deposited on Ti substrate. However, the deposition efficiency was enhanced after adding the carbon nanotube into the deposition processes. As a result, hydrous ruthenium oxide can be deposited on Ti substrate in short deposition period. Fig.3 depicts the electrical capacity characteristics of this specimen. 
[1] H.-S Hwang, Master Thesis, Chung-Hua University, 2004.

[2] Yuli Lin and H.-S Hwang, Microscopy and Microanalysis, Vol. 11(suppl.2), p1756-1757(2005).

[3] This research was supported by NSC94-2216-E-216-009, CHU94-2216-E-216-009 and CHU94-TR-09.
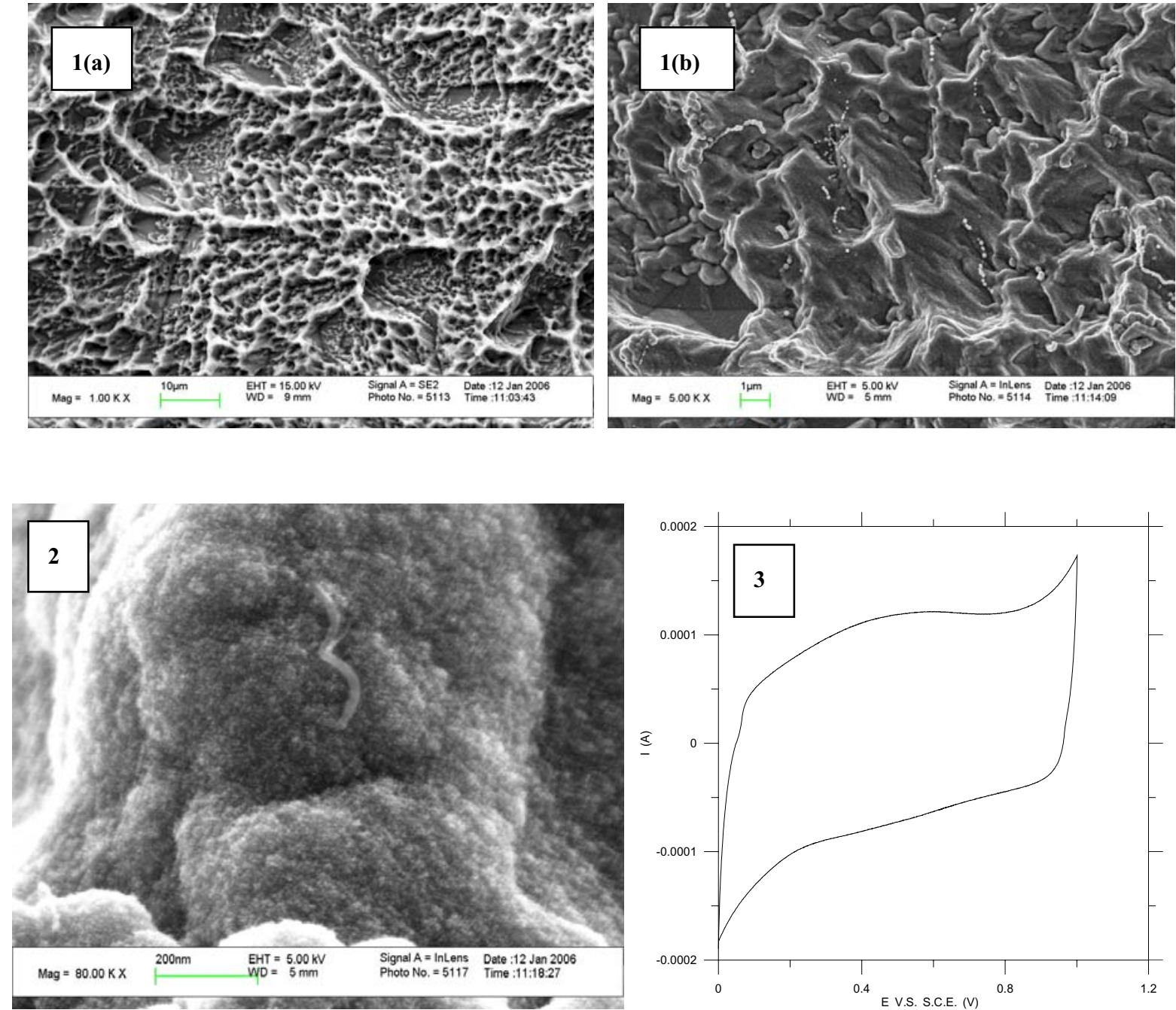

Figure 1. (a) Microstructure of hydrous ruthenium oxide coating with carbon-nanotube additive in the deposition process.

(b) Microstructure of hydrous ruthenium oxide coating with adding carbon-nanotube in the deposition process in higher magnification from (a).

Figure 2. Microstructure of hydrous ruthenium oxide coating with carbon-nanotube additive in the deposition process in higher magnification.

Figure 3. The electrical capacity characteristics of specimens. 